\title{
Meine Höhlenexkursionen im kroatischen Montangebiet.
}

\section{(Col.)}

\section{Von Victor Stiller, Agram.}

Wer von Agram nach Fiume schon mit der Eisenbahn gefahren ist, dem werden die schönen Landschaftsbilder, die er während der Fahrt gesehen hat, gewifs in Erinnerung geblieben sein.

Besonders die Strecke von Moravica bis Fiume zeigt ein eigenartiges prächtiges Bergland: den kroatischen Karst.

Überwiegend nackter Kalkstein, bedeckt ihn doch auf weite Flächen uralter Tannenwald, unter dessen Baumriesen Dunkelheit herrscht. Wald und sonnenbeschienene blumige Alpenwiesen wechseln mit rauhen, trostlos scheinenden Steinwüsten, zwischen deren weifsen Felsblöcken hier und da die rotbraune Eisenoxyderde zu sehen ist, die dem stellenweise kalten Ton der weifsleuchtenden Steine ein wärmeres Gepräge verleiht. Zwar haben wir hier keine von ewigem Schnee bedeckten Riesen, auch keine Gletscher, und erreicht unser Karst kaum die durchschnittliche Höhe von $800 \mathrm{~m}$, dafür fühlen wir aber den unendlichen Reiz und die Eigenart des Karstgebirges mit seinen abwechselnd düster erhabenen wie zarten und lieblichen Landschaften, welche selbst den Tiroler Dolomiten und der Schweiz an Anziehungskraft nicht viel nachgeben.

Die eigenartigen Formationen des Karstes, seine zerrissen aussehenden unwegsamen Steinfelder mit den trichterförmigen Einsenkungen sind ebenso fesselnd als die Gewohnheiten der Bewohner.

Aber nicht nur im grünen Sommer-, auch im Winterkleid ist der Karst hinreifsend schön. Im dichten Schneepelz, aus dem die dunklen Tannen wie verstohlen hervorgucken, sieht er entzückend aus. Das sieht vermutlich auch „Boreas", und mit übermälsiger Freude und stürmisch dringt er auf ihn ein. Da beginnt ein wüstes Treiben! Die Leute sagen "Bora“ und flüchten in die Häuser; die Tannen aber beeilen sich ihre Schneehauben abzuschütteln und müssen nun all ihre Widerstandskraft aufbieten, um im wilden Spiel nicht entwurzelt zu werden. Es werden Häuser abgedeckt, Eisenbahnzüge aus den Schienen gehoben. Da geht's gar herzhaft $\mathrm{zu}$ - es ist kein Ding für Schwächlinge!

Nach einigen Tagen lälst die Kraft des eisigen Windes jedoch nach, und wir sehen den Karst verjüngt und schöner als je! $\mathrm{Oh}$ ! Wie möchte ich ihn besingen, mit Poesie umweben - aber ach!

„Die Sprache ist schön und an Formen so reich, und doch kommt sie nie dem Gedankengang gleich!" Ich weilte viele, viele 
Tage in diesen Bergen, die mir in der kurzen Zeit, seitdem ich sie zum ersten Male sah, schon so fest ans Herz gewachsen sind, und erduldete mit Gleichmut alle damit verbundenen Unbequemlichkeiten. Denn, dafs es bei so viel Licht auch Schatten geben mufs, ist doch selbstverständlich!

Die Herberge ist im ganzen kroatischen Karst ebenso schlecht als Speise und Trank. Freunde eines guten Tropfens sind gar oft gezwungen $\mathrm{zu}$ dursten oder sich nach frischem Quellwasser umzusehen. Aber auch das murmelt nur in Liedern und Romanen an jeder Berglehne. Im Karst kommt es überhaupt kaum vor, und man trinkt dort in der Regel das noch niemals besungene, also höchst prosaische Zisternenwasser.

Abgesehen von all den bekannten Strapazen jeder Gebirgstour, mufs man in unseren Kalkalpen all seinen lieben Gewohnheiten und Bequemlichkeiten entsagen. Nur wer dies imstande ist, bei dem der Drang ins Freie die Bequemlichkeitsliebe überwiegt, kann den Karst geniefsen, sein Sinnen und Fühlen der herrlichen Natur dort widmen!

Für den Entomologen hat der Karst ganz besonderes Interesse. Ist er doch vor allem die Heimat geheimnisvoller Höhlenkäfer. - Zarte Tiere, augenlos, die, abgeschlossen von der Oberwelt, ihr Leben in tief unter der Erdoberfläche gelegenen Höhlen, in ewiger Finsternis, bei stets gleichbleibender Temperatur verbringen, und sich unter diesen langweiligen "finstern" Verhältnissen doch ebenso glücklich und zufrieden fühlen, wie ihre Verwandten auf der sonnenbeschienenen Oberwelt.

Solche Höhlen haben wir im Karst zahlreich; und wo mir Zeit und Umstände es nur halbwegs erlaubten, suchte ich sie auf.

Was mir da schon alles passierte - das mürste ich wohl bei anderer Gelegenheit erzählen, denn es hätte schier kein Ende. Auch fürchte ich, dals meine Frau so Kunde von meinen halsbrecherischen Exkursionen erhalten, und mir dieselben einstellen könnte. Da gab es unvorhergesehene lawinenartige Rutschpartien, dafs mir Hören und Sehen verging; einmal stürzte hinter mir ein Teil der Höhle ein. Und wie oft stiefs ich mir den Kopf an die harten Tropfsteine! Doch Schwamm drüber! Ich kam alleweil - von einigen ungefährlichen Beulen abgesehen - mit heiler Haut wieder ans Tageslicht.

Wer diese Szenerie meiner entomologischen Ausflüge: die Wälder im Karst und seine unterirdische Welt, die Tropfsteinhöhlen, noch nicht gesehen hat, kann sich davon wohl keine rechte Vorstellung machen. Trotzdem will ich es versuchen, auf schriftlichem Wege Führer zu sein, und lade alle Freunde der freien schönen Natur, alle, die für die hier aufgetürmten steinernen 
Wahrzeichen ferner Vergangenheit, sowie für den blühenden und singenden Werdegang auf unsrer Erde Sinn und Verständnis haben, ein, mich im Geiste auf einigen meiner Ausflüge zu begleiten, und eröffne den Reigen mit der

\section{,Eishöhle bei Lokve".}

Es ist eine der kleinen, aber höchst malerisch gelegenen Höhlen Kroatiens, deren tiefster runder Teil für die Senkungsoder Einsturztheorie als Beispiel dienen könnte.

Die Benennung "Eishöhle " ist jedenfalls eine irrige. Denn in der Höhle selbst, respektive deren aphotischen Zone, woselbst die Temperatur sozusagen ständig ist und zwischen +6 bis $+10^{\circ} \mathrm{R}$ schwankt, sind Eisgebilde selbst im strengsten Winter nicht zu bemerken. Es dürfte zu dieser Bezeichnung wohl der Umstand beigetragen haben, dafs der tiefgelegene Höhleneingang bis in die Sommermonate Mai-Juni hinein noch mit gewaltigen Schnee- und Eismassen verlegt ist. Aber eben diese schützende Schneedecke ist es wieder anderseits, welche die verhältnismälsig kleine Höhle vor dem Eindringen der Kälte schützt, ihren gleichmäfsigen Wärmegrad sichert und somit jede Eisbildung im Inneren hindert.

Der Zugang zur Höhle durch „Golubinyak“, der Abstieg von der Eisenbahnstation und der Weg weiter bis zur Höhle ist unbeschreiblich schön und für jeden, besonders für den Naturforscher, der mit vollem Herzen und verständnisklarem Blick die Naturschönheiten wohl doppelt erfafst, unvergefslich!

Die kerzengeraden kolossalen Tannen, die aus Stein so mächtig emporragen, die halb in Moos gebetteten Felsblöcke von abenteuerlichster Gestaltung und dazu das geheimnisvolle grüne Dunkel und die majestätische Ruhe des Waldes: der Eindruck erfaîst die Seele des Wanderers gar gewaltig, dort fühlt er die Allmacht der weltbildenden Urkraft wohl lebhafter als je.

Der Entomologe findet hier alle seine Erwartungen übertroffen; denn unter jedem Stein, die massenhaft herumliegen, unter der Rinde der gestürzten modernden Hölzer, an den knorrigen Wurzeln und meterdicken Stämmen der Bäume, auf Blättërn und Blüten wie am Moose der weifsen Kalkfelsen, findet er seine Lieblinge in reicher, auserlesener Gesellschaft.

Carabus violaceus v. arurescens, Carabus convexus, Cychrus attenuatus, Harpalus brevicollis, Licinus Hoffmannseggi, Molops striolatus, ovipennis und plitoicensis, Nebria Dahli v. litoralis, Trechus croaticus, Byrrhus luniger und gigas, Otiorrhynchus v. punctatissimus, perdix, lutosus, Acalles hypocrita, echinatus und abstersus, Rhynchites hungaricus, Orestia Hampei, Bythinus sculptifrons, Euconnus oblongus, Plintus an- 
ceps, Orina speciosissima usw. gehören $\mathrm{zu}$ den gewöhnlichsten Erscheinungen.

Auf Blüten und Knospen treiben bunte Schmetterlinge ihr freundliches Spiel; besonders beobachtete ich viele Vertreter der Zygaena- und Vanessa-Form. Den schwerfälligen grofsen Parnassius Apolló sieht man hier seltener. Um so häufiger fliegt er beiläufig $80 \mathrm{~m}$ höher, bei der Bahnstation Lič z. B., wo er $-880 \mathrm{~m}$ Meereshöhe - in den Sommermonaten zu den gewöhnlichsten Erscheinungen zählt.

Die Caraben und Carabiciden meiner Sammlung stammen zum überwiegend gröfsten Teil aus der herrlichen Gegend LokveDelnice, wo ich sie teilweise sogar im Winter aus morschen Baumstämmen oder unter Moos hervorholte.

Seltener begegnete ich Carabus croaticus, Kreutzeri und variolosus, Ophonus incisus, Cymnidis coardunata und axillaris, Laemostenus elongatus, Molops simplex und longipennis, Saphanus piceus, Cercylon semistriatum usw., doch auch diese Arten erbeutete ich gelegentlich meiner Wanderung zur Höhle schon einige Male.

Die in manchen Gegenden des Karstes so häufige und mit Recht gefürchtete $\mathrm{Vip}$ e ra a modite ${ }^{1}$ ) kommt hier, respektive in der Umgebung der Höhle so selten vor, dafs sie für den Entomologen keine nennenswerte Gefahr bildet. Trotzdem würde ich raten, beim Umwälzen der Steine, unter denen sich oft die seltensten Coleopteren aufhalten, nur den Stock oder Schirm und die Füfse zu gebrauchen, um die blofsen Hände der Gefahr des Gebissenwerdens nicht so sehr auszusetzen.

Da ich vordem die im Winter gefundenen Caraben erwähnte, kann ich nicht unterlassen, die eigentümliche Stellung des Carabus Kreutzeri im Winterquartier zu erwähnen, um so mehr, als ich mich nicht erinnere, je darüber etwas gelesen zu haben.

In einer kugelrunden Höhlung vom Durchmesser der Käferlänge, unter der Rinde morschen Nadelholzes „kniet“ der Käfer, so dafs ich den ersten, den ich so fand, für eine grofse Spinne hielt. Erst mit der Pinzette herausgezogen, erkannte ich in ihm C. Kreutzeri. Er war noch nicht ausgefärbt und machte den Eindruck eines Schwächlings. Zu meinem Vergnügen jedoch fand ich Ende November v. J. ein vollentwickeltes schönes Exemplar gegenwärtig der schönste $C$. Kreutzeri meiner Sammlung - unter ganz gleichen Umständen.

Mein damaliger Versuch, das runde Nest auszuschneiden, mifsglückte infolge des schon ganz morschen weichen Materials. Auf Schritt und Tritt findet der Entomologe Interessantes,

1) Nashornviper. 
keine Rede ist. Die Höhle ist trotzdem sehr feucht, ohne Luftzug, mit nur einem Ausgang und beherbergt eine besonders an Coleopteren reiche Fauna.

Ich fand daselbst: Anophthalmus Bilimeki likanensis, A. hirtus Stilleri Ganglb , Bathyscia acuminata, sowie typische Formen des Leptoderus Hohenvarti, Astagobius angustatus und Propus sericeus.

Leptoderus und Astagobius, deren eigentümlicher Körperbau mit den langen zarten Fühlern und Beinen sie so recht zum Leben im Finstern und infolge ihres schmalen augenlosen Kopfes und langen Prothorax zum Durchforschen der engsten Steinritzen befähigt, ist hier aufserordentlich häufig. So zwar, dafs sich die Gefangenen, die oft weit mehr als den Boden des Fangglases bedecken, mit den Lockspeisen: Käse, Schneckenfleisch und Schwämmen, trotzdem ich, um dies zu verhindern, immer einige Stückchen Papier ins Glas gebe, einen schmutzigen Knäuel bilden und sich gegenseitig beschädigen.

Anophthalmus und Bathyscia hingegen, wie auch Propus kommt seltener vor. Anophthalmus B. likanensis habe ich hier nur dreimal, A. hirtus Stilleri Ganglb. fünfmal gefangen. Von Bathyscia acuminata fand ich bisher 3 Stück, davon eines in der Vorhöhle.

An sonstigen Arthropoden wären Titanethes, Brachydesmus, Campodea und Obisium zu erwähnen. Dieselben sind jedoch nicht genauer determiniert und stehen in den wenigen Exemplaren, die ich noch besitze, Reflektanten zur Verfügung.

Alle diese genannten Arten bewohnen die Höhle gleichmälsig verteilt. So fand ich z. B. Leptoderus und Astagobius in den hintersten, verstecktesten Teilen der aphotischen Zone ebenso in Gesellschaft von Anophthalmus und Bathyscia, wie in der dem Eingang schon näheren, vom Licht erreichten Dämmerzone. Allerdings überschreitet keine von ihnen eine gewisse Dunkelheitsgrenze und konnte ich in der Vorhöhle ihrer niemals habhaft oder ansichtig werden - eine schnellfüfsige, wahrscheinlich dahin verirrte Bathyscia ausgenommen. Dies hängt jedenfalls mit der Temperaturdifferenz zusammen, denn vom Licht können die in Rede stehenden "Augenlosen" schwerlich abgehalten werden.

Gegen höhere Temperatur sind alle höhlenbewohnenden Arthropoden aufserordentlich empfindlich. In den kühlen Abendstunden aus der Höhle gebracht, zeigen sie noch keine besondere Unruhe und halten sich über Nacht im Freien gelassen ganz gut. Anders benehmen sie sich jedoch bei Tage der Heimat entführt, wenn in den Sommermonaten der Temperaturunterschied über $15^{0}$ beträgt. Nervöses, hastiges Umherrennen, Aufdenrückenfallen, zuckende Bewegung der Fühler und Beine usw., kurz, wir sehen dann das- 
selbe Bild des Todeskampfes, wie bei einem in Weingeist geworfenen Käfer, nur dem Wärmegrad entsprechend verlängert. Ein in die heifse Mittagssonne gestellter Anophthalmus likanensis aus der Touiner Höhle war sozusagen momentan tot und rührte sich nicht mehr, während sein im Schatten des Höhleneinganges kurz vorher in Spiritus gegebener Kamerad noch Lebenszeichen von sich gab.

Da ich aus den überfüllten Fanggläsern der Lokveer Höhle von besonders erwünschten Arten, wie Anophthalmus und Bathyscia, oft nur mehr einzelne Körperteile zu Gesicht bekam, versuchte ich den Andrang durch entsprechende Verteilung der Lockspeise hintanzuhalten, indem ich in ein Glas z. B. nur Käse, in ein anderes nur schimmlige Blätter usw. gab, und hoffte so die gefräIsige Gesellschaft zu trennen. Aber weit gefehlt! Alles wird angenommen und keine Art ist wählerisch. Am gewöhnlichsten fressen sie sich wahrscheinlich gegenseitig auf.

Dafs die zarten Sylphiden die stets kampfbereiten und wohlbewehrten Anophthalmus-Arten angreifen, kann ich wohl nicht zugeben - selbst wenn sie in grofser Übermacht sind. Dafs sie diese jedoch auffressen und zum mindesten das Abdomen ausweiden, sobald sie durch irgend einen Umstand wehrlos wurden, steht aufser Zweifel - denn das haben mir ihre Überreste in den Fanggläsern leider oft genug erzählt.

Die Tropfsteinbildung der Lokveer Höhle ist eine geringe. Von den gewaltigen Stalaktiten und Stalakmiten anderer Grotten und Höhlen im Karst ist hier nicht viel zu sehen. Kaum dafs man an den Wänden schwache Tropfsteingebilde wahrnimmt. Jedenfalls beeinträchtigt dieser Mangel die Schönheit dieses sonst so fesselnden Stückchens Unterwelt nicht wenig.

Schliefslich will ich noch den Umstand erwähnen, dafs ich trotz häufigen Vorkommens der Tiere nur ein einziges Mal ein am Boden kriechendes Astagobius-Pärchen erblickte, ansonsten aber trotz fleifsigen Suchens und sogar Siebeversuchen im Geröll nichts „Lebendes" finden konnte. Dazu gehören jedenfalls sehr scharfe Augen. Nach dem ersten, dieserart in entomologischer Hinsicht ganz erfolglosen Besuch der „Eishöhle“ war ich sogar der Meinung, dafs sie „unbewohnt" sei. Bei späterem Nachdenken leuchtete mir jedoch die Unwahrscheinlichkeit dieser Annahme ein, und ich wiederholte den Versuch mit Fanggläsern. Der Erfolg war ein glänzender; so zwar, dafs ich überzeugt bin, nun alle ihre Bewohner zu kennen, trotzdem die Möglichkeit durchaus nicht ausgeschlossen ist, dafs einzelne unbemerkt gebliebene Arten die angebotene Lockspeise denn doch verschmähten, ansonsten auch sehr selten sind oder nur zu gewissen Zeiten auftreten. 
Ich besuchte die Höhle schon einige Male; da sie den Vorteil gut gangbaren Zuganges besitzt und da mir darin auch niemals Böses widerfuhr, erinnere ich mich ihrer stets mit vielem Vergnügen. Nur einmal - es war vor 3 Jahren - störte mein Vergnügen ein anderer Höhlenbesucher, der sich jedoch nicht sehen lassen wollte und mir mit seiner Unsichtbarkeit beinahe Angst einjagte.

Der Höhleneingang war nämlich von grofsen Schneemassen noch verlegt und nur ein schmaler Spalt war schon frei. Ich versuchte durch diesen hinein $\mathrm{zu}$ gelangen. Da sah ich auf dem Schnee die ganz frischen Spuren eines Mannes mit landesüblichen Sandalen, die vor mir direkt in die Höhle führten. Da ein anderer Ein- oder Ausgang nicht besteht und keine Spuren herausführten, mufsten wir nun 2 in der Höhle sein. Erst schaute ich herum, aber das schwache Kerzenlicht reicht nicht weit. Die herumliegenden grofsen Steinblöcke mit von der Nähe grell beleuchteten Flächen und dem anstofsenden Kernschatten fast ohne Übergang, dabei die von den Lichtstrahlen nicht mehr erreichte gähnende Finsternis dahinter, weisen jeden neugierigen Blick entschieden zurück.

Ich rief also in der Landessprache: Cuješ, molim, idite napred, ne bojse! - keine Antwort. Nur die von Zeit zu Zeit herabfallenden Wassertropfen unterbrachen die Totenstille.

Ich leugne nicht, dafs mir etwas bange zumute war. Aber gezeigt habe ich es dem andern nicht. Nach Höhlenbewohnern spähend und Steine wälzend, ging ich bis ans Ende der Höhle, kam langsam wieder zurück, und ich wette, der andere hat in seinem Versteck vor Erstaunen über mein ihm unverständliches Beginnen während der Zeit den Mund offen gehalten. Vielleicht hat's ihm auch gegruselt. Denn abergläubisch sind die Leute einmal und im alten Reiseüberzieher mit aufgeschlagenem Kragen, den treuen Regenschirm unterm Arm, ein Glas und die funkelnde Pinzette in der Hand, dabei in stark vorgebeugter Haltung mit der Kerze nach Käfern spähend, hat er mich vielleicht für den alten Berggeist „Rübezahl“ gehalten?!

Genug an dem, ich verliefs die Höhle ohne Unfall und liefs den andern unten, ohne ihm "Lebewohl" zuzurufen.

Ein seliges Gefühl ist es immer, wenn man aus der nafskalten Finsternis wieder ins warme Sonnenlicht tritt. Man möchte aufjauchzen im Grufs des Lichtes!

Beim Ordnen und Betrachten der aus der Höhle herausgebrachten Beute kommen dann die verschiedensten Gedanken. Wie war ihre noch "sehende" Ahnenform? Wie geschah die An- 
passung? Welcher Zusammenhang besteht zwischen ihnen, dafs die Arten, die doch die Grenzen ihres engen Höhlenheims nie überschreiten, an weit auseinander gelegenen Orten so oft die ganz gleichen sind? Welche Ursache bedingt die blasenartig aufgetriebene Körperform der Leptoderini? Wie leben und ernähren sie sich?

In Fanggläsern machte ich - wie schon gesagt - oft die Erfahrung, dafs die Carabicidae besonders nicht nur anderen Arten, sondern auch ihren eigenen schwächeren Kameraden und Gattungsgenossen das Abdomen abfressen. Kopf mit daranhängenden Brustringen und Deckflügeln bleibt, das Abdomen ist herausgefressen. Kein Zweifel also :

Tief unter der Erde finden unter den kleinen augenlosen Geschöpfen gewils ebenso erbitterte Kämpfe auf Leben und Tod statt, wie im Leben allwegs. Der Starke frifst den Schwächeren seit jeher, immer und überall. Wie seinerzeit der schreckliche Lelaps den riesengrofsen, aber ziemlich wehrlosen Brontosaurus. zerrifs, so nährt sich auch heute der mit mörderischen Kiefern ausgerüstete Anophthalmus zum Teil von den schwächeren Mitbewohnern der Höhle, sei es in Larven- oder Imagogestalt. Es ist eben der vielgenannte Kampf ums Dasein. Selbst da, wo nach unserem Ermessen fürs "Sein" beinahe alle Bedingungen fehlen, finden wir noch reiches organisches Leben mit all seinen Wünschen, Begierden und Kämpfen.

Aber allen Kämpfen zum Trotz und dem Entomologen zum Trost verderben und verschwinden die Arten nicht so bald, entwickeln sich vielmehr um so kräftiger und widerstandsfähiger, damit den alten Spruch bestätigend:

„Das Leben siegt!"

\section{Zur Kenntnis der Arctiidengattung Anaxita Wlk. (Lep.)}

\section{Von Embrik Strand, Berlin (Kgl. Zoolog. Museum).}

Die zentral- und südamerikanische Arctiidengattung Anaxita Wlk. fehlt in $\mathrm{Hamps}$ an s Monographie dieser Familie (Cat. Lep. Phal. Br. Mus., Vol. III), und zwar, weil er sie für eine Hypside hält, nach Exemplaren aus der ehemaligen Staudingerschen Sammlung zu urteilen, die von Hampson bestimmt und als Hypsiden bezeichnet worden sind. In Übereinstimmung mit früheren Autoren, die über diese Tiere geschrieben haben, ist es mir gänzlich uner- 


\section{$2 \mathrm{BHL}$ Biodiversity Heritage Library}

Stiller, Victor. 1911. "Meine Höhlenexkursionen im kroatischen Montangebiet. (Col.)." Deutsche entomologische Zeitschrift 1911(4), 467-475.

https://doi.org/10.1002/mmnd.48019110417.

View This Item Online: https://www.biodiversitylibrary.org/item/103379

DOI: https://doi.org/10.1002/mmnd.48019110417

Permalink: https://www.biodiversitylibrary.org/partpdf/236076

\section{Holding Institution}

Harvard University, Museum of Comparative Zoology, Ernst Mayr Library

\section{Sponsored by}

Biodiversity Heritage Library

\section{Copyright \& Reuse}

Copyright Status: Public domain. The BHL considers that this work is no longer under copyright protection.

This document was created from content at the Biodiversity Heritage Library, the world's largest open access digital library for biodiversity literature and archives. Visit BHL at https://www.biodiversitylibrary.org. 